\title{
Random Forest Model Prediction of Compound Oral Exposure in the Mouse
}

Haseeb Mughal ${ }^{1}$, Han Wang ${ }^{2}$, Matthew Zimmerman², Marc d. Paradis ${ }^{3}$, and Joel S. Freundlich ${ }^{1,4, *}$

1. Department of Pharmacology, Physiology, and Neuroscience, Rutgers University - New Jersey Medical School, Newark, New Jersey, 07103, USA.

2. Center for Discovery and Innovation, Hackensack Meridian Health, Nutley, New Jersey, 07110, USA.

3. Holdings \& Ventures, Northwell Health, Manhasset, New York, 11030, USA

4. Division of Infectious Disease, Department of Medicine and the Ruy V. Lourenço Center for the Study of Emerging and Re-emerging Pathogens, Rutgers University - New Jersey Medical School, Newark, New Jersey 07103, USA 


\section{Contents}

Table S1. R ${ }^{2}$ and RMSE statistics for initial random forest models with cross-validation and external test sets with randomly partitioned data.

Table S2. $\mathrm{R}^{2}$ and RMSE statistics for random forest models created with a feature selection loop using partitioning by linear sampling.

Table S3. $\mathrm{R}^{2}$ and RMSE statistics for random forest models created with a feature selection loop using partitioning by linear quartiles sampling.

Table S4. $\mathrm{R}^{2}$ and RMSE statistics for random forest models created with a feature selection loop using partitioning by random quartiles sampling.

Figure S1. Random forest workflow metanode information: Data preprocessing.

Figure S2. Dataset partition scheme.

Figure S3. Random forest workflow metanode information: Cross-validation.

Figure S4. Plot of distribution of values in the dataset for $\mathrm{AUC}_{0-5 \mathrm{~h}}$.

Figure S5. Random forest workflow metanode information: Quartile processing.

Supporting File 1: Supporting File 1_FeaturesAndModels.xlsx

Supporting File 2: Supporting File 2_SnapshotPKworkflow.knwf

Supporting File 3: Supporting File 3_SnapshotPKdataset.csv 
Table S1. $\mathbf{R}^{2}$ and RMSE statistics for initial random forest models constructed through feature optimization with cross-validation and external test sets with randomly partitioned data. For each random seed, a 60/20/20 split of the data was achieved and a random forest model was constructed with the training set and then used to predict the cross-validation and external test sets.

\begin{tabular}{|c|c|c|c|c|c|}
\hline Random Seed & $\begin{array}{c}\mathrm{R}^{2} \\
\text { (cross-validation) }\end{array}$ & $\begin{array}{c}\mathrm{RMSE} \\
\text { (cross-validation) }\end{array}$ & $\begin{array}{c}\mathrm{R}^{2} \\
\text { (external test) }\end{array}$ & $\begin{array}{c}\text { RMSE } \\
\text { (external test) }\end{array}$ & $\begin{array}{c}\text { Model } \\
\text { Number }\end{array}$ \\
\hline 1594346895338 & 0.811 & 0.336 & 0.792 & 0.662 & 1 \\
\hline 1598068012413 & 0.653 & 0.550 & 0.292 & 0.452 & $\mathrm{n} / \mathrm{a}$ \\
\hline 1598068236021 & 0.506 & 0.890 & 0.094 & 0.343 & $\mathrm{n} / \mathrm{a}$ \\
\hline 1598220100706 & 0.413 & 0.600 & 0.597 & 0.652 & $\mathrm{n} / \mathrm{a}$ \\
\hline 1598068446029 & 0.822 & 0.441 & 0.870 & 0.325 & $\mathrm{n} / \mathrm{a}$ \\
\hline
\end{tabular}


Table S2. $\mathbf{R}^{2}$ and RMSE statistics for random forest models created with a feature selection loop using partitioning by linear sampling. Models $2-6$ were the top five models from the maximization of $\mathrm{R}^{2}$ for the cross-validation set in the feature selection loop. Models $7-11$ were the top five models from the minimization of RMSE for the cross-validation set in the feature selection loop. Furthermore, $\mathrm{R}^{2}$ and RMSE were obtained from a ten-fold cross-validation with the entire dataset.

\begin{tabular}{|c|c|c|c|c|c|c|}
\hline $\begin{array}{c}\text { Model } \\
\text { number }\end{array}$ & $\begin{array}{c}\text { Cross- } \\
\text { validation/ } \\
\text { external test set }\end{array}$ & $\mathrm{R}^{2}$ & RMSE & $\begin{array}{c}10 \text {-fold } \\
\text { cross- } \\
\text { validation } \\
\mathrm{R}^{2}\end{array}$ & $\begin{array}{c}10 \text {-fold } \\
\text { cross- } \\
\text { validation } \\
\text { RMSE }\end{array}$ & $\begin{array}{c}\text { Number of } \\
\text { features }\end{array}$ \\
\hline \multirow[t]{2}{*}{2} & Cross-validation & 0.961 & 0.257 & \multirow[t]{2}{*}{0.478} & \multirow[t]{2}{*}{0.720} & \multirow[t]{2}{*}{26} \\
\hline & External test set & 0.737 & 0.819 & & & \\
\hline \multirow[t]{2}{*}{3} & Cross-validation & 0.961 & 0.258 & \multirow[t]{2}{*}{0.481} & \multirow[t]{2}{*}{0.718} & \multirow[t]{2}{*}{27} \\
\hline & External test set & 0.736 & 0.819 & & & \\
\hline \multirow[t]{2}{*}{4} & Cross-validation & 0.961 & 0.258 & \multirow[t]{2}{*}{0.478} & \multirow[t]{2}{*}{0.720} & \multirow[t]{2}{*}{25} \\
\hline & External test set & 0.739 & 0.818 & & & \\
\hline \multirow[t]{2}{*}{5} & Cross-validation & 0.960 & 0.258 & \multirow[t]{2}{*}{0.485} & \multirow[t]{2}{*}{0.715} & \multirow[t]{2}{*}{33} \\
\hline & External test set & 0.736 & 0.811 & & & \\
\hline \multirow[t]{2}{*}{6} & Cross-validation & 0.960 & 0.259 & \multirow[t]{2}{*}{0.486} & \multirow[t]{2}{*}{0.715} & \multirow[t]{2}{*}{32} \\
\hline & External test set & 0.737 & 0.811 & & & \\
\hline \multirow[t]{2}{*}{7} & Cross-validation & 0.959 & 0.273 & \multirow[t]{2}{*}{0.491} & \multirow[t]{2}{*}{0.712} & \multirow[t]{2}{*}{39} \\
\hline & External test set & 0.819 & 0.783 & & & \\
\hline \multirow[t]{2}{*}{8} & Cross-validation & 0.959 & 0.274 & \multirow[t]{2}{*}{0.489} & \multirow[t]{2}{*}{0.713} & \multirow[t]{2}{*}{52} \\
\hline & External test set & 0.818 & 0.781 & & & \\
\hline \multirow[t]{2}{*}{9} & Cross-validation & 0.959 & 0.274 & \multirow[t]{2}{*}{0.490} & \multirow[t]{2}{*}{0.713} & \multirow[t]{2}{*}{51} \\
\hline & External test set & 0.818 & 0.781 & & & \\
\hline \multirow[t]{2}{*}{10} & Cross-validation & 0.959 & 0.274 & \multirow[t]{2}{*}{0.490} & \multirow[t]{2}{*}{0.713} & 50 \\
\hline & External test set & 0.818 & 0.781 & & & \\
\hline 11 & Cross-validation & 0.959 & 0.274 & 0.490 & 0.713 & 49 \\
\hline & External test set & 0.818 & 0.781 & & & \\
\hline
\end{tabular}


Table S3. $\mathbf{R}^{2}$ and RMSE statistics for random forest models created with a feature selection loop using partitioning by linear quartiles sampling. Models $12-16$ were the top five models from the minimization of RMSE for the cross-validation set in the feature selection loop. Models $17-21$ were the top five models from the maximization of $\mathrm{R}^{2}$ for the cross-validation set in a feature selection loop. $\mathrm{R}^{2}$ and RMSE were obtained from a ten-fold cross-validation with the entire dataset.

\begin{tabular}{|c|c|c|c|c|c|c|}
\hline $\begin{array}{l}\text { Model } \\
\text { number }\end{array}$ & $\begin{array}{c}\text { Cross- } \\
\text { validation/ } \\
\text { external test set }\end{array}$ & $\mathrm{R}^{2}$ & RMSE & $\begin{array}{c}10 \text {-fold } \\
\text { cross- } \\
\text { validation } \\
\mathrm{R}^{2}\end{array}$ & $\begin{array}{c}10 \text {-fold } \\
\text { cross- } \\
\text { validation } \\
\text { RMSE }\end{array}$ & $\begin{array}{c}\text { Number of } \\
\text { features }\end{array}$ \\
\hline \multirow[t]{2}{*}{12} & Cross-validation & 0.836 & 0.491 & \multirow[t]{2}{*}{0.613} & \multirow[t]{2}{*}{0.624} & \multirow[t]{2}{*}{20} \\
\hline & External test set & 0.730 & 0.557 & & & \\
\hline \multirow[t]{2}{*}{13} & Cross-validation & 0.840 & 0.494 & \multirow[t]{2}{*}{0.612} & \multirow[t]{2}{*}{0.625} & \multirow[t]{2}{*}{21} \\
\hline & External test set & 0.734 & 0.555 & & & \\
\hline \multirow[t]{2}{*}{14} & Cross-validation & 0.848 & 0.512 & \multirow[t]{2}{*}{0.620} & \multirow[t]{2}{*}{0.618} & \multirow[t]{2}{*}{24} \\
\hline & External test set & 0.776 & 0.531 & & & \\
\hline \multirow[t]{2}{*}{15} & Cross-validation & 0.849 & 0.512 & \multirow[t]{2}{*}{0.620} & \multirow[t]{2}{*}{0.619} & \multirow[t]{2}{*}{25} \\
\hline & External test set & 0.776 & 0.531 & & & \\
\hline \multirow[t]{2}{*}{16} & Cross-validation & 0.848 & 0.512 & \multirow[t]{2}{*}{0.620} & \multirow[t]{2}{*}{0.618} & \multirow[t]{2}{*}{23} \\
\hline & External test set & 0.775 & 0.532 & & & \\
\hline \multirow[t]{2}{*}{17} & Cross-validation & 0.895 & 0.668 & \multirow[t]{2}{*}{0.622} & \multirow[t]{2}{*}{0.623} & \multirow[t]{2}{*}{49} \\
\hline & External test set & 0.788 & 0.555 & & & \\
\hline \multirow[t]{2}{*}{18} & Cross-validation & 0.895 & 0.668 & \multirow[t]{2}{*}{0.622} & \multirow[t]{2}{*}{0.622} & \multirow[t]{2}{*}{48} \\
\hline & External test set & 0.788 & 0.555 & & & \\
\hline \multirow[t]{2}{*}{19} & Cross-validation & 0.895 & 0.668 & \multirow[t]{2}{*}{0.622} & \multirow[t]{2}{*}{0.622} & \multirow[t]{2}{*}{47} \\
\hline & External test set & 0.788 & 0.550 & & & \\
\hline \multirow[t]{2}{*}{20} & Cross-validation & 0.895 & 0.668 & \multirow[t]{2}{*}{0.617} & \multirow[t]{2}{*}{0.627} & 50 \\
\hline & External test set & 0.789 & 0.555 & & & \\
\hline 21 & Cross-validation & 0.895 & 0.635 & 0.636 & 0.609 & 31 \\
\hline & External test set & 0.793 & 0.556 & & & \\
\hline
\end{tabular}


Table S4. $\mathbf{R}^{\mathbf{2}}$ and RMSE statistics for random forest models created with a feature selection loop using partitioning by random quartiles sampling. Models $22-26$ were the top five models from the maximization of $\mathrm{R}^{2}$ for the cross-validation set in the feature selection loop. Models $27-$ 31 were the top five models from the minimization of RMSE for the cross-validation set in a feature selection loop. $\mathrm{R}^{2}$ and RMSE were obtained from a ten-fold cross-validation with the entire dataset.

\begin{tabular}{|c|c|c|c|c|c|c|}
\hline $\begin{array}{l}\text { Model } \\
\text { number }\end{array}$ & $\begin{array}{c}\text { Cross- } \\
\text { validation/ } \\
\text { external test set }\end{array}$ & $\mathrm{R}^{2}$ & RMSE & $\begin{array}{c}10 \text {-fold } \\
\text { cross- } \\
\text { validation } \\
\mathrm{R}^{2}\end{array}$ & $\begin{array}{c}10 \text {-fold } \\
\text { cross- } \\
\text { validation } \\
\text { RMSE }\end{array}$ & $\begin{array}{c}\text { Number of } \\
\text { features }\end{array}$ \\
\hline \multirow[t]{2}{*}{22} & Cross-validation & 0.916 & 0.483 & \multirow[t]{2}{*}{0.508} & \multirow[t]{2}{*}{0.699} & \multirow[t]{2}{*}{21} \\
\hline & External test set & 0.486 & 0.646 & & & \\
\hline \multirow[t]{2}{*}{23} & Cross-validation & 0.916 & 0.487 & \multirow[t]{2}{*}{0.509} & \multirow[t]{2}{*}{0.700} & \multirow[t]{2}{*}{22} \\
\hline & External test set & 0.499 & 0.637 & & & \\
\hline \multirow[t]{2}{*}{24} & Cross-validation & 0.916 & 0.479 & \multirow[t]{2}{*}{0.519} & \multirow[t]{2}{*}{0.692} & \multirow[t]{2}{*}{20} \\
\hline & External test set & 0.488 & 0.644 & & & \\
\hline \multirow[t]{2}{*}{25} & Cross-validation & 0.915 & 0.488 & \multirow[t]{2}{*}{0.508} & \multirow[t]{2}{*}{0.700} & \multirow[t]{2}{*}{23} \\
\hline & External test set & 0.498 & 0.637 & & & \\
\hline \multirow[t]{2}{*}{26} & Cross-validation & 0.914 & 0.486 & \multirow[t]{2}{*}{0.509} & \multirow[t]{2}{*}{0.699} & \multirow[t]{2}{*}{24} \\
\hline & External test set & 0.494 & 0.641 & & & \\
\hline \multirow[t]{2}{*}{27} & Cross-validation & 0.928 & 0.324 & \multirow[t]{2}{*}{0.553} & \multirow[t]{2}{*}{0.668} & \multirow[t]{2}{*}{21} \\
\hline & External test set & 0.624 & 0.547 & & & \\
\hline \multirow[t]{2}{*}{28} & Cross-validation & 0.927 & 0.324 & \multirow[t]{2}{*}{0.549} & \multirow[t]{2}{*}{0.671} & \multirow[t]{2}{*}{24} \\
\hline & External test set & 0.613 & 0.555 & & & \\
\hline \multirow[t]{2}{*}{29} & Cross-validation & 0.927 & 0.324 & \multirow[t]{2}{*}{0.549} & \multirow[t]{2}{*}{0.671} & \multirow[t]{2}{*}{23} \\
\hline & External test set & 0.613 & 0.555 & & & \\
\hline \multirow[t]{2}{*}{30} & Cross-validation & 0.927 & 0.325 & \multirow[t]{2}{*}{0.552} & \multirow[t]{2}{*}{0.669} & 20 \\
\hline & External test set & 0.616 & 0.553 & & & \\
\hline 31 & Cross-validation & 0.927 & 0.325 & 0.549 & 0.671 & 22 \\
\hline & External test set & 0.611 & 0.557 & & & \\
\hline
\end{tabular}


Figure S1. Random forest workflow metanode information: Data preprocessing.

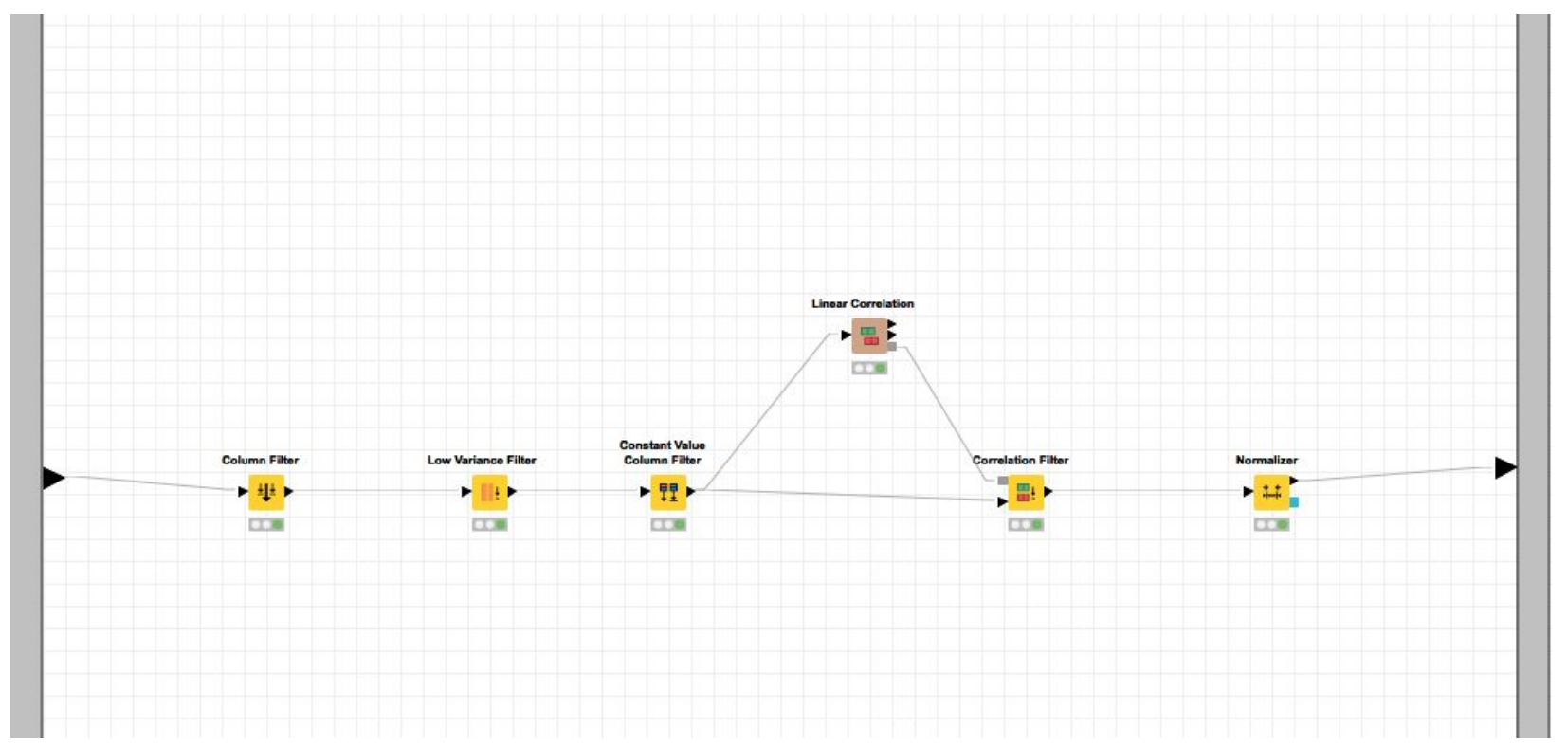


Figure S2. Dataset partition scheme.

\section{Snapshot PK assay dataset}

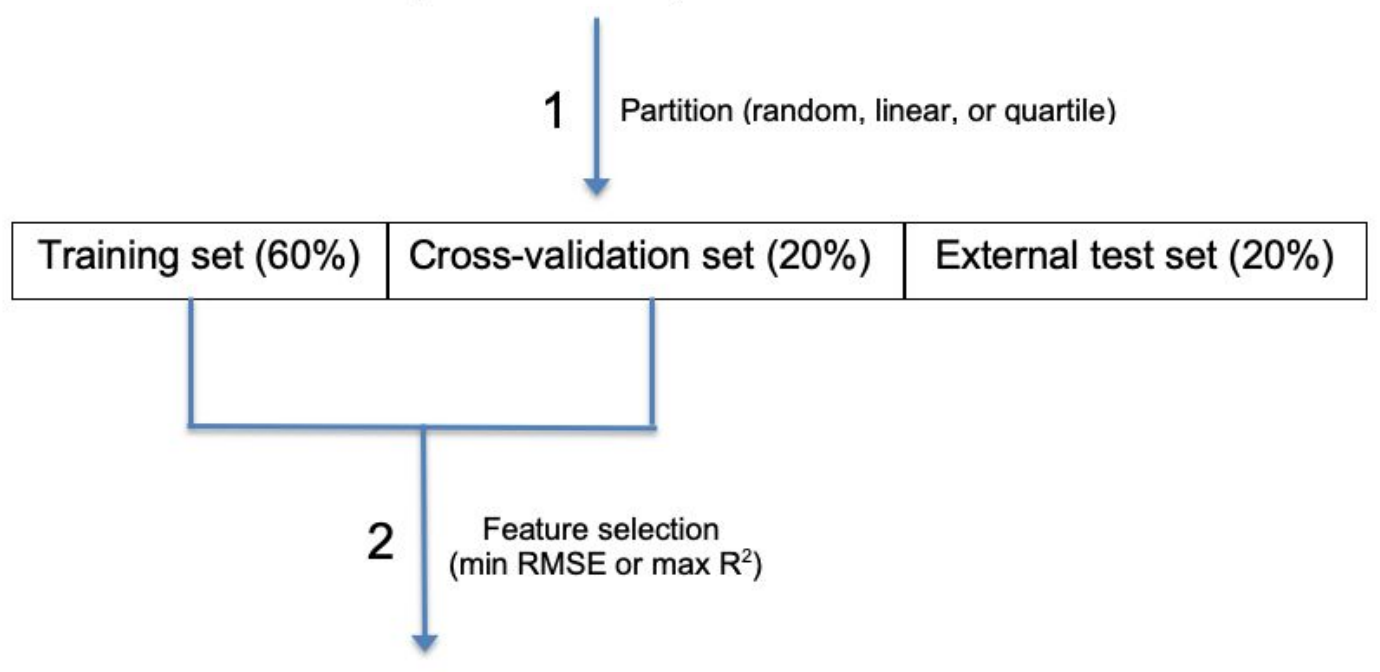

\section{Top 31 models}

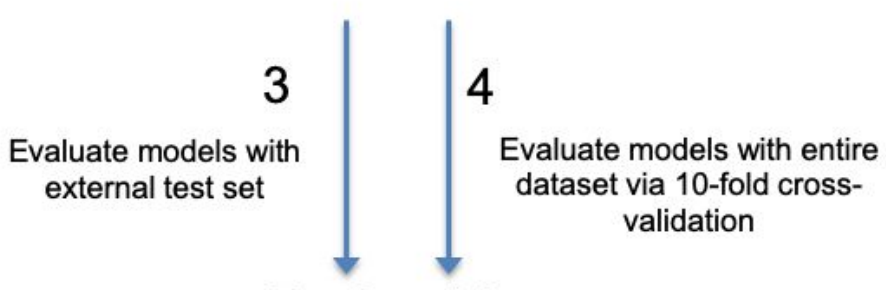

Top 9 models 
Figure S3. Random forest workflow metanode information: Cross-validation.

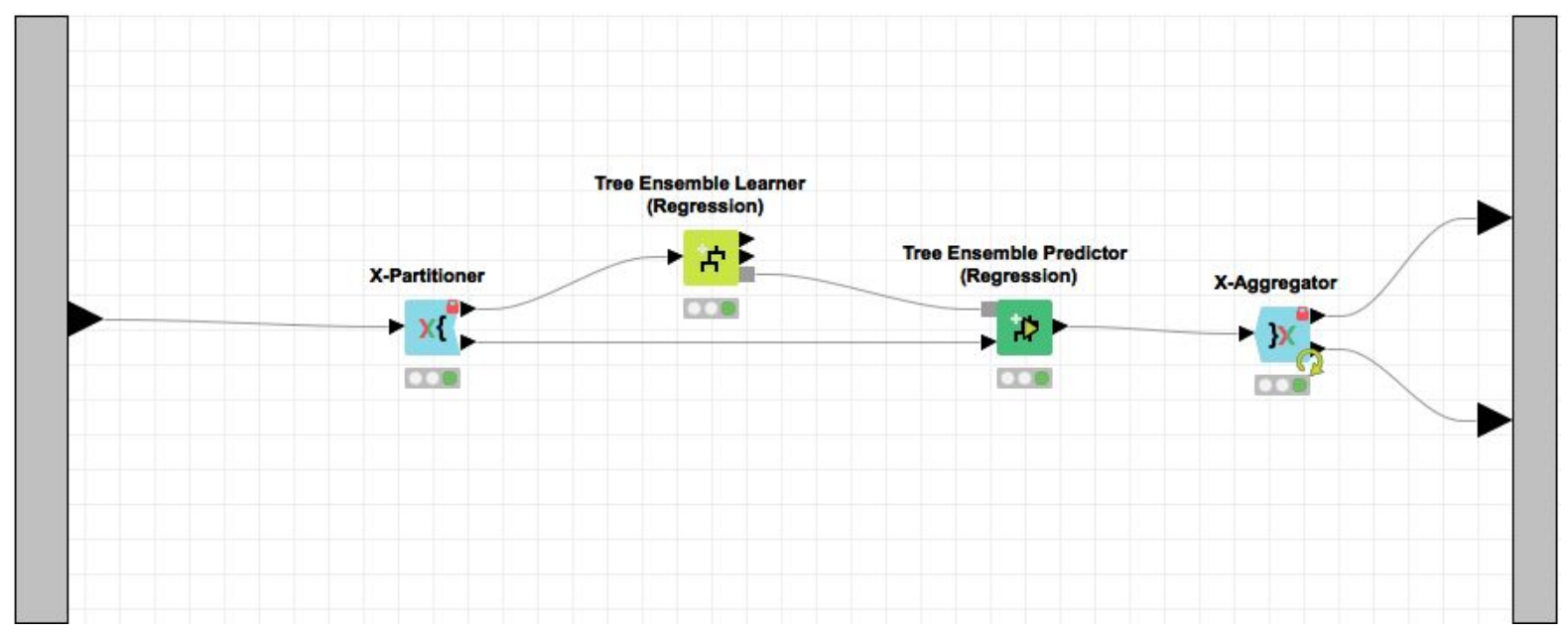


Figure S4. Plot of distribution of values in the dataset for $\mathbf{A U C}_{\mathbf{0 - 5 h}}$. Microsoft Excel (Vers. 16.4.2) was utilized to divide the range of $\mathrm{AUC}_{0-5 \mathrm{~h}}$ into quartiles and then calculate the frequency of entries in each quartile.

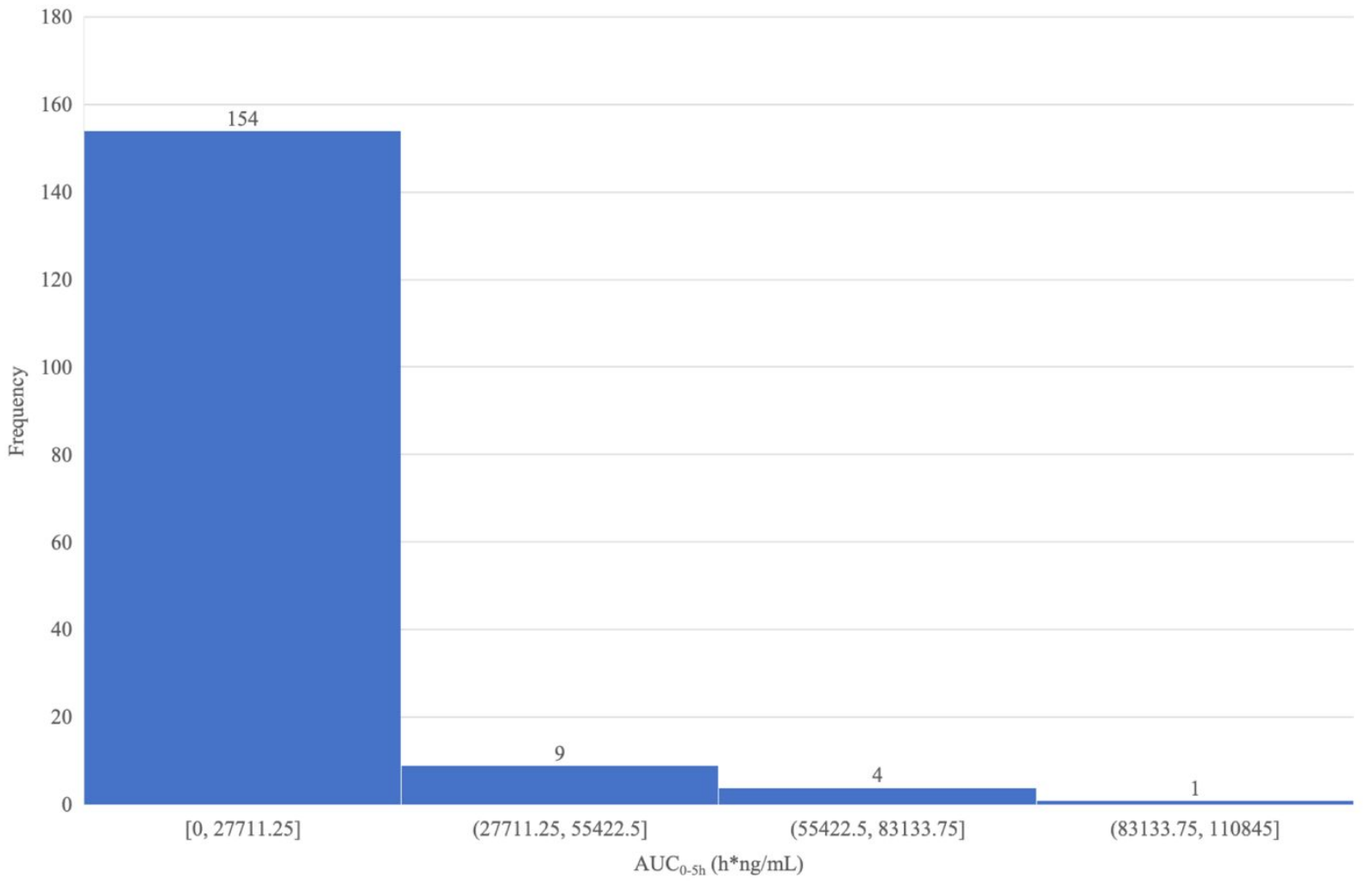


Figure S5. Random forest workflow metanode information: Quartile processing.

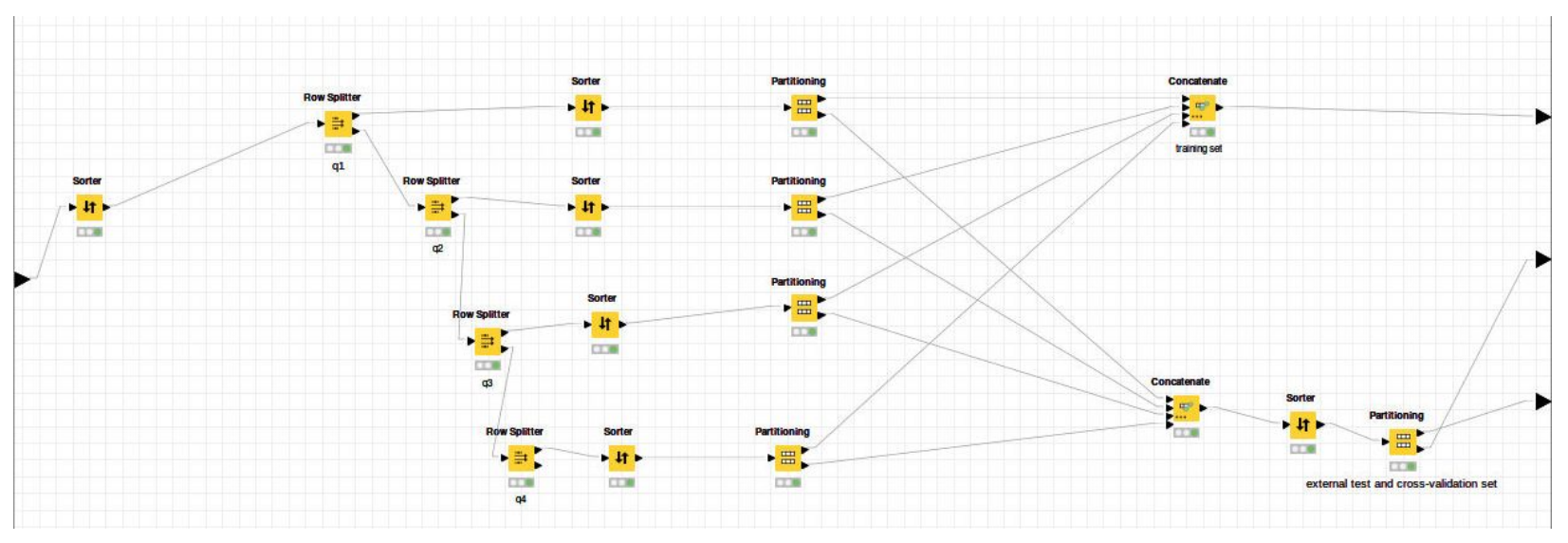

